\title{
Processing demands during mental operations
}

\author{
BETH KERR* \\ University of Oregon, Eugene, Oregon 97403
}

\begin{abstract}
Man possesses a central system of limited capacity. Theorists at first described this system as a single limited capacity channel. Two current theoretical alternatives to single-channel theory are (1) the undifferentiated capacity hypothesis that man possesses a pool of capacity units so that interference occurs only if the total number of capacity units that mental operations demand exceeds the system limit and (2) the hypothesis that some, but not all, mental operations require space in a limited capacity central mechanism and that any operation that requires space will interfere with any other operation that also demands space. Time on task fails as a sensitive measure of capacity demands because some task components require time but not full processing capacity. The secondary task technique uses the interference between a primary task and a secondary task to assess the extent to which the primary task makes processing demands on the central limited system. Processing demands have been measured for five categories of mental operation: (1) encoding, (2) multiple input, (3) rehearsal, (4) transformation, and (5) responding.
\end{abstract}

One stage in the development of a scientific theory involves the specification of systems in terms of boundary conditions or limits (Feynman, 1965). The specification of boundary conditions may provide a theoretical framework within which one can search for a unified set of laws. Over the past 30 years, the study of human limitation in processing signals has been a focal point for the development of psychological theory.

The notion that humans possess a central system of limited capacity arises both from subjective experience and from man's frequent inability to perform two tasks simultaneously (James, 1890; Broadbent, 1971). Presumably, there is some type of limited capacity mechanism which is required in the performance of most voluntary acts. While there are a number of different theories on the nature of the limited system, they all have in common the assumption that under some conditions two signals which simultaneously require access to the limited capacity system will interfere with each other. Interference is a reduction in the efficiency of processing a signal that is measured by changes in the speed or accuracy of responses to the signal.

It is possible to determine the extent to which any pair of tasks requires the limited capacity system by comparing performance on each task attempted individually to performance on tasks attempted together. Whenever performance on a primary task that is combined with a secondary task is equivalent to control performance on the primary task alone, scores on the secondary task may be used to measure the degree to which the primary task requires the limited capacity system. Thus, one way of describing a task or mental operation is in terms of the demands it places on the limited capacity system, as measured through secondary task performance.

Processing any signal may require a number of different mental operations. Although it may not be

*I would like to thank Michael Posner and Steven W. Keele for their suggestions and comments on earlier drafts of this manuscript. This paper was written while the author was supported by a NIMH predoctoral research fellowship. possible to isolate and study each specific mental operation, some distinct categories of mental operations are commonly associated with signal processing. For convenience, this paper deals with five such broadly defined classes of mental operations which can be distinguished experimentally from one another. These operations are (1) encoding, (2) multiple input, (3) rehearsal, (4) transformation, and (5) responding. The first section of the paper reviews the theoretical interpretations of the nature of the limited capacity system. The second section reviews the uses of the secondary task interference technique as a tool for measuring processing demands. The final section compares and contrasts the extent to which different mental operations require the limited capacity central mechanism and examines the influence of various task parameters on processing demands.

\section{THEORETICAL ISSUES}

\section{Limited Capacity Theories}

\section{Single-Channel Theory}

The concept of a limited capacity system was advanced in the 1950s in response to the growing body of literature relating reaction time to amount of information transmitted and to stimulus-response compatibility. The notion that response decisions require time in a central limited capacity system replaced the concept of independent associative links between every stimulus and its appropriate response. Theorists at first viewed the human operator as a single limited capacity channel (Welford, 1952; Broadbent, 1958). A signal was thought to occupy the entire channel from the time it was selected until a response could be initiated. Whenever two signals were presented, one signal captured the entire channel. A second or unselected signal could be filtered or held in store and permitted to enter the channel only after the response to 
the first signal had been completed. The total time for task completion or the time increase per unit of transmitted information served as measures of the processing demands a signal placed on the limited capacity system.

\section{Steps Toward Alternatives to Single-Channel Theory}

Further research prompted theorists to modify the single-channel hypothesis. A crucial finding was that with extensive practice (Seibel, 1963; Mowbray \& Rhoades, 1959) or highly compatible stimulus-response assignments (Leonard, 1959) response times did not increase with the number of alternatives. This result suggested either the paradoxical idea of a channel with infinite capacity or a return to the assumption of independent stimulus-response linkages. However, as both of these alternatives proved unattractive, theorists began to question (1) the use of time as a measure of processing demands and (2) the notion that all components of task performance require exclusive use of a limited channel.

The single-channel hypothesis equates the time needed to process a signal with the time and extent that the signal places demands upon the limited capacity system. However, time alone fails as a sensitive measure of capacity demands, because it does not allow for the idea that some task components require time but not full processing capacity. Task interference studies provide a means for separating reaction time from demands on the limited capacity system. Whenever two tasks must be performed simultaneously or in close succession, the time delays over control performance on each separate task alone provide clues to the extent to which tasks require access to a limited capacity system.

Results from task interference studies strengthened the evidence that alternatives for the single-channel hypothesis were needed. The single-channel hypothesis predicts that the time to perform two different tasks together will equal (serial processing) or exceed (serial processing with recovery time) the sum of the time required to perform each task alone. This is often the case. However, in some instances the time to perform a pair of tasks is much less than the sum of the time to complete each by itself (e.g., Keele, 1967). Thus, it is clear that in some cases task components are processed in parallel and do not require exclusive use of a single channel.

As a rule, psychological refractory period studies, which require separate responses to two signals presented in close succession, provide results consistent with single-channel theory. The response to the second signal is delayed until the response to the first signal has been completed (Bertelson, 1966). However, studies that manipulate the difficulty of the second signal suggest that more overlap occurs as the number of alternative responses in the second task increases. An example is Karlin and Kestenbaum's Experiment II (1968), in which Ss received a signal for a two-choice visual task followed by a signal for either a one- or two-choice auditory task. The response to the auditory task was always delayed. However, the delay for the two-choice signal was only slightly longer than the delay for the one-choice signal and was much shorter than the delay single-channel theory would predict from the difference between performance on the one- and two-choice auditory tasks performed alone (see Keele, 1973, for a discussion). Some mental operations associated with the difficult second task were processed in parallel with the first task.

Thus, evidence from a number of sources points out the need to modify single-channel theory to allow parallel processing. Two theories that allow parallel processing have been proposed. Both theories consider the extent to which operations require space in the limited system rather than temporal limitations or the rate at which information passes through the system. ${ }^{1}$ One theory "widens" the single channel to allow mental operations performed on more than one signal to share processing space. One theory "shortens" the channel to a mechanism necessary to some but not all mental operation.

\section{Undifferentiated Capacity Hypothesis}

The first theory is that man possesses some capacity maximum, so that interference occurs only when the total processing capacity that tasks demand exceeds the system limits. Each operation is said to draw from a library of undifferentiated processing units, with all demands met in full as long as the library has the required units in supply. Results from practiced Ss and for compatible input-output systems led Moray (1967) to propose a limited capacity processor, analogous to a time-sharing computer. Processing space was allocated as needed to the operations performed on the signal rather than to the signal itself. Kahneman (1973) extended this line of argument, suggesting a capacity model that views man as possessing a pool of "effort" that may be allocated to tasks. Both the extent of the effort pool and the allocation policy are influenced by internal and external factors such as arousal effects and dispositions reflecting rules for selective attention. ${ }^{2}$ Interference is nonspecific and depends upon the total demands made by the tasks competing. for the capacity units available rather than specific operations within any one given task.

\section{Limited Capacity Central Mechanism Hypothesis}

The second theory is that some, but not all, mental operations require space in a single limited capacity central mechanism (Posner \& Keele, 1970). Any mental operation that requires the limited capacity central mechanism interferes with a second mental operation that simultaneously requires the mechanism. In this case, 
each operation that requires processing capacity makes demands upon a library which supplies in full only one patron at a time. Some theorists have, in effect, shortened the single channel to a mechanism associated primarily with responding (Deutsch \& Deutsch, 1963; Norman, 1968). Others feel that a number of mental operations require space in the limited capacity central mechanism (Posner \& Keele, 1970; Keele, 1973). In either case the point is made that, while certain mental operations always require the mechanism, other mental operations never require the mechanism and may proceed in parallel with other operations without interference.

\section{Distinguishing Between Hypotheses}

Both the undifferentiated total capacity hypothesis and the hypothesis that specific mental operations always require space in a limited capacity central mechanism allow that different operations will demand varying degrees of processing capacity. Thus, the hypotheses are difficult to test experimentally. At present, no strong evidence is available to support one theory over the other. A study showing that two tasks (A and B) interfere in separate tests with a third task (C) and do not interfere with each other would support the undifferentiated capacity hypothesis over the limited capacity central mechanism hypothesis. This situation could occur under the undifferentiated capacity hypothesis if Task A and Task B each require slightly less than half the available capacity and time share with each other without interference and Task $\mathrm{C}$ requires almost all available capacity and interferes with both $\mathrm{A}$ and $\mathbf{B}$. To date, evidence of this sort is not available. Situations in which two tasks each interfere with a third task and with each other could occur under both hypotheses. However, some support for the limited capacity central mechanism position comes from evidence (presented later in this paper) that encoding and memory look-up do not require central processing capacity and may proceed in parallel with other operations without interference, while multiple input, rehearsal, transformation, and responding operations consistently cause interference.

\section{Describing the Limited Capacity Central Mechanism}

Attempts to describe the hypothetical limited capacity central mechanism lead to further questions which help clarify the operation of the mechanism.

\section{Range of Interference}

One question is whether only similar operations interfere or whether interference is more widespread (Peterson, 1969). Studies using a secondary discrete simple reaction-time task presented strategically within different primary task components show that a number of primary task operations, in addition to responding, interfere with responding to the secondary task (e.g., Johnston, Griffith, \& Wagstaff, 1972a; Posner \& Keele, 1970). Interpolated tasks inserted between short-term memory list presentation and recall lead to reduced item recall. Here interpolated task operations, such as responding or transformation, interfere with the short-term memory rehearsal operation (e.g., Posner \& Rossman, 1965). Thus, it appears that a mental operation that requires processing capacity will tend to interfere with many operations that also require processing capacity. Recent studies have suggested, however, that the degree to which operations interfere may depend on the extent to which they place overlapping demands on specific subsystems, such as the verbal system or the spatial system (e.g., Brooks, 1967; Allport, Antonis, \& Reynolds, 1972).

\section{Serial or Parallel Processing}

The limited capacity central mechanism may be viewed as either a short single channel that operates serially or as a parallel system that processes multiple signals with reduced efficiency. With a serial processor, the delay for a secondary task would depend solely upon the time the primary mental operation required the mechanism. With a parallel processor, the delay for a secondary task might depend on both the amount of time and the proportion of the space the primary mental operation required in the mechanism.

Since either a serial processor or a processor operating in parallel with interference would cause time delays in a second mental operation, distinguishing between these two ideas is difficult. Posner (personal communication) has suggested that it may be possible to distinguish between a parallel processor and a serial processor by using a primary task with stages known to produce different amounts of interference, combined with two secondary tasks that require different amounts of processing capacity. If the processor operates in serial fashion, so that each mental operation that requires space occupies the mechanism completely for a period of time, the interference with the more difficult secondary task will be longer than that with the easier secondary task by a constant amount across all stages of the primary task. However, with a parallel processor, the primary task stages requiring more processing capacity would allow less parallel processing and cause greater interference with the more difficult secondary task than with the easier secondary task. For the secondary task scores, an interactive effect would support a parallel processor and an additive effect would support a serial processor. The secondary tasks for such a study would have to be selected carefully, since the more difficult secondary task must require more processing capacity. not merely more execution time, than the easier task.

Since interference indicates a demand on processing 
capacity with either a serial or parallel processor, the distinction is not critical to the central issue of determining which mental operations require processing capacity.

\section{SECONDARY TASK TECHNIQUE}

\section{Historical Perspective}

At the end of the 19th century, psychologists began to ask Ss to attempt to perform two tasks at once. Binet (1890) reported a study intended to measure the hypothetical general ability to divide attention by comparing ability to press a tube in rhythm while reading aloud, reciting learned passages, or performing mental arithmetic. In this same vein, Jastrow (1891-1892) studied different types of tapping tasks paired with reading aloud and mental arithmetic. The notion that mental operations might be studied by comparing their degree of interference with a common secondary task was advanced by the biologist Jacques Loeb. Loeb's student, Welch (1898), used the ability to maintain a standard hand pressure as a common secondary task during arithmetic, counting, and reading tasks. Although a few early 20th century studies continued to use divided attention techniques, both divided attention and secondary task studies soon fell into disuse with the rise of behaviorism. Studies requiring $\mathrm{Ss}$ to perform two simultaneous tasks reappeared following World War II and paved the way for the reemergence of the secondary task technique within the last 15 years.

A number of other studies requiring performance on two tasks preceded secondary task technique studies. These studies documented the human S's ability to attempt two simultaneous tasks and paved the way for the notion that scores on one task may be used to measure processing demands for another task. Studies employing the filler technique developed by Brown (1958) and Peterson and Peterson (1959) suggested that processing demands for a filler task, such as counting backward by $3 \mathrm{~s}$, prevented short-term memory rehearsal (see Crowder, 1964, for a discussion). Interpolated task studies were used to force performance differences between two levels of a primary task that proved equal without secondary task requirements (e.g., Garvey \& Taylor, 1959) and to test practice effects (e.g., Bahrick, Noble, \& Fitts, 1954). Success with interpolated tasks prompted the utilization of a concomitant secondary task to measure processing during unimpaired performance on a primary task. Early work compared overall processing demands for two tasks without pinpointing specific mental operations. For example, Brown and Poulton (1961) compared secondary memory and classification task performance during car driving in residential areas and shopping areas. Drivers made more errors on the secondary tasks in shopping as opposed to residential areas, implying that shopping area driving requires more processing capacity. However, the specific aspects of shopping area driving that require more processing capacity are not defined. Studies extending the secondary task technique to isolate specific mental operations are products of only the last 5 or 6 years.

\section{Description}

The secondary task technique requires that Ss perform a second task in order to assess processing demands during a primary task. The secondary task should be designed so that it does not affect primary task performance. Ss are aware that they are to respond to the second task without allowing the response to interfere with the primary task. When the secondary performance is poorer than control performance on the secondary task alone, the primary task is said to require processing capacity. Continuous tasks such as tracking, discrete tasks such as reaction time to press a key in response to a tone or light flash, and signal detection tasks have been used as secondary tasks. The increase in error scores or reaction times above control performance on the secondary task indicates the degree of interference caused by the primary task and reflects the processing demands of the primary task. Performance on secondary tasks may be used to compare processing demands during the component mental operations that comprise a primary task. For example, scores on a secondary task may be used to evaluate the processing demands during encoding, rehearsal, and responding in a primary task such as letter matching. Performance on the secondary task may also be compared for two levels of the primary task. For example, in rehearsing long as opposed to short word lists, secondary scores reflect the relative degree of processing capacity required for the two lists.

\section{Methodological Issues}

The secondary task technique has proven a successful tool for isolating and comparing processing demands. However, a few general reservations should be raised before interpreting secondary task scores.

\section{Detecting Small Processing Demands}

The problem of distinguishing between serial or parallel with interference processing invites the criticism that the secondary task technique fails to detect small processing demands. When no decrement in secondary task performance occurs, one may reason either that the primary task does not require processing space or that the primary task requires so little processing space that sufficient space remains for the secondary task to be processed in parallel (Kahneman, 1973). This objection, however, is not fatal, since processing scores may be compared along a relative scale. Studies that show that a 
particular mental operation does not interfere with either an easy or a difficult secondary task are required to show that a mental operation does not require processing capacity. If a mental operation requires low amounts of processing in a parallel processor, it should be possible to increase the difficulty of the secondary task until it no longer can be processed in parallel with the primary task.

\section{Changes in Primary Task Performance Due to the Secondary Task}

Since the secondary task is intended to measure processing demands during a primary task, it is essential that Ss direct their attention toward the primary task rather than switch or alternate attention between the two tasks. Scores on the primary task in the dual task setting should equal scores on the primary task in a control situation without the secondary task. When these scores differ, results are often difficult to interpret, since secondary task scores cannot be considered a pure measure of processing during normal primary task performance. Certain experimental designs discourage switching more successfully than others. Mental operations, such as rehearsal, which do not demand immediate overt responses would seem most apt to allow switching. It also appears that $\mathrm{Ss}$ are more likely to switch between a continuous secondary task and the primary task than they are to switch between a discrete secondary task and the primary task. Investigators often discourage switching by establishing payoffs that guarantee higher awards for the primary task (e.g., Johnston et al, 1972a). Ss prove amazingly versatile and adjust easily to payoff modifications. Fortunately, it appears that performance is optimal when Ss are paid to devote full attention to the primary task rather than employ a strategy maximizing combined performance on both tasks (Kahneman, 1970).

Even when Ss "protect" the primary task, showing equal control and dual performance scores, and receive payoffs biased toward primary task performance, critics argue that the requirement to perform two simultaneous tasks changes normal processing demands. Welford (1968), for example, suggested that basic channel capacity may increase with the requirement to perform two tasks, especially if the tasks are speeded. In contrast, Kahneman (1973) argued that maintaining a divided set and expecting a second signal, in and of themselves, require processing capacity, leaving less capacity for regular task demands. These restrictions would seem less stringent with tasks structured so that a discrete secondary task probe occurs on only a proportion of the trials at varying points within the primary task (e.g., Posner \& Keele, 1970). It is acknowledged that in all cases Ss are aware that they must perform more than one task and may not face the same processing demands required in the single-task setting. However, secondary scores collected within one given paradigm certainly reflect relative processing demands for varying levels of the primary task at different points during primary task performance.

\section{Structural Interference}

It is essential to distinguish interference caused by demands for space in a central processing mechanism from interference caused by structural limitations. Structural interference is thought to occur whenever two tasks place incompatible or excessive demands on specific perceptual, memory, or response systems (Kahneman, 1973). Needless to say, a $S$ cannot be expected to receive simultaneously two widely separated visual signals or to perform two responses with the same hand. The problem, of course, is determining what constitutes the limits of structural interference. Shallice (1972) has defined a series of "effector units" for which tasks must compete. An effector unit could, for example, be unspecific to the extent of including the execution of all overt physical responses. The difficulties encountered in producing and monitoring more than one rhythm suggest that rhythm maintenance may also require a single effector unit for which tasks compete. Spatial representation appears to be limited whenever common effector units are necessary for visual tasks. Since structural interference may occur when the primary and secondary tasks compete for the same effector units, both structural and capacity interference may be present within a given paradigm. An additional consideration is that different secondary tasks may interact differentially with a given primary task or different primary tasks may interact differentially within one secondary task. Thus, it again appears that processing demands are most accurately assessed within one specific paradigm using the same secondary task to evaluate various levels or points within one primary task. Within one paradigm, the structural interference should remain more constant, allowing the comparison of relative processing demands across the primary task.

\section{Evaluating Processing Demands at Probed Positions}

A final question concerns the extent to which scores for a discrete secondary task probe reflect processing demands at the instant in the primary task that the probe occurs. Responding to a probe requires a period of time. Processing demands for the primary task may change in this period that follows secondary probe onset. Thus, secondary task scores may reflect primary processing demands at secondary signal onset, primary processing demands at some time following signal onset, or compounded demands throughout the period. Thus, when primary task demands change rapidly, secondary scores may show a large interference effect that is difficult to interpret. This is often the case when a primary task signal requires an immediate response. For example, in the typical psychological refractory period 
study, two signals separated by a short amount of time call for immediate responses. Delayed second responses in this situation reflect processing demands associated with the time period during the primary task that includes signal identification, response selection, and response initiation. Which specific task components require processing capacity cannot be easily determined. The same problem may occur when the primary task is a movement to be executed as quickly as possible. Investigators often avoid the use of secondary task probes that reflect more than one primary task mental operation by choosing naturally segmented primary tasks that do not require immediate responses throughout. A two-letter successive-stimulus same-different matching task, for example, does not allow the $S$ to respond until the second stimulus has been presented. List learning and recall, a primary task, is naturally divisible into separate encoding, rehearsal, and responding stages. Although the restriction that the primary task fall into natural segments restricts the use of the secondary task technique, the interference measure is more meaningful when it can be associated with a specific operation within the primary task.

\section{PROCESSING DEMANDS DURING MENTAL OPERATIONS}

\section{Introduction}

The secondary task technique has been employed to study processing demands in five categories of mental operation: encoding, multiple input, rehearsal, transformation, and responding. Encoding is defined as the operations required as one stimulus item is received and contacts its representation in memory. Multiple input, however, entails a series of operations associated with receiving a group of stimuli, such as a list of words or a sentence. In this case, one must both encode one stimulus and retain or comprehend the meaning of previously presented items. Rehearsal refers to mental operations that "maintain" an item or list of items in memory for later use. In the cases reviewed, the rehearsal period is not subject to other primary task demands. During multiple-item tasks, rehearsal may be confounded with encoding, transformation, and responding, since Ss must retain all items while operating on individual items. The specific processing demands for rehearsal in these instances have not been separated experimentally from processing demands made by other operations. Transformations include operations requiring some type of mental manipulation of stimulus information, for example, rearranging word lists into alphabetic order or combining two words during an impression formation task. Responding encompasses a number of operations associated with "producing" answers in a variety of settings, such as recall tasks or tasks requiring the execution of a discrete or continuous motor response. Of course, the extent to which these five categories of operations can be distinguished from each other depends upon the experimental designs that have been employed. Rehearsal, as noted above, is often confounded with another operation and at times is confounded with two additional operations, for example, encoding and transformation in multiple-item lists (e.g., Kahneman, Beatty, \& Pollack, 1967). An attempt is made to isolate these specific mental operations, though occasionally less precise distinctions have been allowed with conflicts noted.

\section{Encoding}

Experimentally it has been difficult to separate from other operations the pure case of encoding as a stimulus is received and contacts memory. In multiple-item lists, Ss encode while concurrently operating on earlier input and expecting further input. Even in the case of single-item input, distinguishing encoding from subsequent rehearsal, transformation, or response selection often proves difficult. Recently, however, Posner and his associates isolated the encoding stage in a same-different letter matching task and measured processing demands with a secondary task requiring a simple reaction time to an auditory tone probe (Posner \& Boies, 1971; Posner \& Keele, 1970; Posner \& Klein, 1973). The S receives a warning signal, followed $500 \mathrm{msec}$ later by the first letter, which in turn is followed $1000 \mathrm{msec}$ later by the second letter. The S uses his right hand to indicate whether the letters are same or different and his left hand to turn off the tones which occur in varying time positions across trials. Reaction times for tones that follow the first letter, as compared to either control tones that occur in the intertrial interval or the lowest tone times across the trial, indicate that encoding the first letter does not require processing. The length of the encoding period has been varied by manipulating the length of the interstimulus interval between the letters or the duration of the first stimulus letter, but in all cases secondary task reaction-time scores during the actual encoding period do not show interference and rise only following the encoding period. Comstock (1972) shortened the duration of the first letter to only $15 \mathrm{msec}$, either with or without a visual mask $100 \mathrm{msec}$ after stimulus onset. Processing demands at stimulus onset remained the same during masked and unmasked trials. This pattern occurred for probes in both a Donders Type $c$ auditory secondary reaction-time task and a simple auditory secondary reaction-time task. The lack of interference during the encoding period holds for nonletter stimuli (Gibson figures) as well as for letters (Posner \& Klein, 1973).

The notion that encoding does not require attention has been supported by studies showing parallel preparation and encoding (Posner \& Boies, 1971) and parallel category activation and encoding (Warren, 1972; Conrad, 1972). The finding that secondary task scores 
remain constant throughout the time interval following presentation of a single item in a multiple-item list also suggests that the actual operation of encoding one stimulus does not increase processing demands (Johnston et al, 1972a). As noted above, however, some may argue that very small processing demands during encoding have not been detected in studies that employ secondary tasks that make relatively low processing demands.

\section{Multiple Input}

The term multiple input is employed here to encompass the operations associated with encoding, retaining, and comprehending a series of items or a sentence as it is being presented. In contrast to single-item encoding, multiple-input operations involve encoding a stimulus item while simultaneously remembering previously presented items. The studies to be reviewed included an initial multiple-input stage, during which several items were presented before any primary task responses were required. The multiple-input stage preceded either a response stage or a pure rehearsal stage that was followed by a response stage.

Processing demands as measured by a secondary task are lower during multiple input than during recall but higher than control performance on the secondary task, indicating that some processing capacity is required during item presentation (Martin, 1970; Trumbo \& Milone, 1971; Johnston et al, 1972a; Johnston, Wagstaff, \& Griffith, 1972b; Johnston, Greenberg, Fisher, \& Martin, 1970). The two studies that have compared multiple input, rehearsal, and recall show that multiple input requires less processing than recall and more processing than rehearsal (Trumbo \& Milone, Experiment II, 1971; Johnston et al, 1970). The evidence for this second comparison of rehearsal and multiple input is less than satisfactory, since the Johnston work compared scores across different experiments and Trumbo and Milone, in contrast to other studies of processing demands during rehearsal, found scores on the secondary task during rehearsal no higher than control performance. However, as multiple input does involve a rehearsal component, in addition to other operations, one might anticipate higher processing demands during multiple input than during rehearsal.

The factors which influence processing demands during multiple input for lists of items tend to be variables associated with the physical nature of the presentation rather than the linguistic or organizational characteristics of the words presented. Secondary tracking performance is worse during fast as opposed to slow presentation rate and is worse in low signal/noise conditions (words presented in loud background noise) than in high signal/noise conditions (words presented without background noise) (Johnston et al. 1970). In contrast, processing demands have not differed for lists with category Words in blocks as opposed to nonblocked lists, nor has processing varied with the association strength of list members (Martin. 1970). Johnston et al (Experiment I, 1972b) did detect lower processing demands for categorized as opposed to uncategorized lists but only with a difficult secondary low-intensity light probe. In this study, the lists were presented for 12 learning trials. Ss learned categorized lists faster than uncategorized lists and may have been able to ignore word presentation and shift emphasis to the detection task on repeated trials of the categorized lists.

Although organizational and linguistic variables rarely influence processing demands during multiple input for word lists, these factors may be determinants of the magnitude of processing demands during sentence comprehension. Foss and Lynch (1969) and Foss (1969) measured processing demands during sentence acquisition by requiring Ss to attend to a secondary task cue embedded within the sentence itself. Ss listened to sentences to be comprehended and, in addition, pressed a button in response to the phoneme $/ b /$ when it occurred during the sentence. Probe reaction times for the phoneme task during sentences that were comprehended correctly were (1) higher when the target word followed a low-frequency as opposed to a high-frequency word, (2) higher when the target occurred early rather than late in the sentence, and (3) higher during self-embedded than right-branching sentences. However, it should be noted that these differences may not be related to interference between sentence comprehension and phoneme identification. Savin and Bever (1970) have suggested instead that phoneme recognition time is related to word recognition. Thus, syntactic complexity may affect the time to identify phonemes because it affects the time to recognize words.

Since each additional item in a multiple-item presentation increases retention-rehearsal load, one might anticipate that processing demands increase across the multiple-input stage. However, differences in processing demands for stages of multiple input vary with the task and methodology employed. Martin (1970) found no difference between secondary tracking errors during early and late input as the $S$ encoded a series of digits or lights. In contrast, Johnston and associates show changes across the input stage with a discrete secondary task. Johnston et al (1972a) found that processing demands increased during normal high signal/noise presentation, with each additional word presented in a task requiring later recall for positional (serial position) or semantically (category name) probed items. Secondary probe reaction times during low signal/noise presentation formed a $U$-shaped function. with the highest processing demands during initial and final list items. Johnston et al (1972b) reported a similar U-shaped function for both another semantically probed list study and a study using a nine-word list presented for free recall. The different secondary task pattems that oceur during input cumnot be linked with list length. 
presentation rate, or type of recall. No consistent pattern emerges. However, the U-shaped function does parallel the classic bowed serial position free recall curve and suggests that initial and final item superiority may be related to the amount of processing capacity these items require.

The literature dealing with changes in processing demands across repeated trials is equally confusing. Martin (1970) found no improvement in secondary tracking scores during input across three repeated serial learning trials. However, Trumbo and Milone (1971) found that secondary tracking errors decreased across five repeated learning trials, and Johnston et al (1972b) found discrete secondary detection scores improved across 12 trials for a free recall list and 18 trials for a semantically probed recall list, with the greatest drop during the first few trials.

\section{Rehearsal}

With one exception (Trumbo \& Milone, Experiment II, 1971), a series of studies employing both tracking (Johnston et al, Experiment II, 1970; Trumbo $\&$ Milone, Experiment I, 1971) and a variety of discrete secondary tasks (Shulman \& Greenberg, 1971; Shulman, Greenberg, \& Martin, 1971; Stanners, Meunier, \& Headley, 1969) showed processing demands during retention and rehearsal. The rehearsal stage follows the presentation of items such as words, a trigram, or a light sequence and precedes a manual or verbal recall stage. Processing demands increase as lists become more difficult to recall. The degree of processing required for rehearsing a list of letters or words is affected by list length, with higher processing demands for long than short lists (Shulman et al, 1971; Johnston et al, 1970), and by item difficulty, with higher processing demands for more difficult items (Stanners et al, 1969). In addition, the degree of interference with the secondary task decreases across the length of the retention period (Shulman \& Greenberg, 1971; Shulman et al, 1971; Stanners et al, 1969). This reduction in processing demands over rehearsal time may reflect a decreased rehearsal load as items are learned and stored in long-term memory.

\section{Transformation}

The secondary task technique has been applied in four very different paradigms which provide evidence that mental transformations requiring Ss to "compute" answers from stimulus material impose processing demands. These demands are higher than pure rehearsal demands and vary with the difficulty of the transform. In these tasks, Ss cannot select their responses by retrieving the correct answer from memory but must combine two stimuli (Diller, 1971), rearrange stimuli (Johnston et al, Experiments III and IV, 1970), or apply some constant mathematical operation (Posner \& Klein,
1973: Kahneman et al, 1967) in order to answer correctly. In this latter mathematical paradigm, Ss with extensive practice on a limited stimulus set may eventually convert to a simpler paired associate task but must initially, at least, apply a mental transformation.

Kahneman et al (1967) devised a primary task in which Ss listened to a string of four digits presented auditorally at one per second, and $1 \mathrm{sec}$ after the last digit recalled at one per second a transformation of the list achieved by adding one to each digit (e.g., 7842 became 8953). As a secondary task, Ss monitored for the letter " $K$ " embedded within a series of letters presented visually at five per second throughout the entire primary task. Detections were reported following each trial. The number of signals missed on the secondary detection task indicated that processing demands increased sharply as each digit was presented and decreased with each digit recalled. Kahneman also recorded pupil diameters for each second of the primary task in a control condition. Pupil measurements paralleled the pattern of the letter detection errors, with diameters increasing with each digit presented and decreasing with each digit recalled. Kahneman and Wolman (reported in Kahneman, 1970) reported the same interference pattern using a different secondary task which required that Ss identify a letter presented for $80 \mathrm{msec}$ during the digit transformation task. Unfortunately, this design does not isolate the primary task transformation stage, since Ss may combine transformation with encoding and rehearsal during digit presentation or with rehearsal and retrieval during the recall stage. A comparison of these secondary task scores with secondary scores during a primary task demanding only the encoding and recall of a four-digit string would be informative. Although comparisons across different paradigms are questionable, it may be mentioned that the steep rise from nearly $20 \%$ to beyond $50 \%$ errors in letter detection during digit presentation in this study differs from the U-shaped pattern during list presentation in the Johnston et al (1972b) study or the slow rise in the Johnston et al (1972a) study, suggesting that transformation imposes an additional processing load during multiple-item presentation.

Posner and Klein (1973) compared secondary task tone reaction-time scores recorded during the same-different letter matching task described above and a letter task transformation with the same time parameters. The transformation task required the $S$ to add three to the first letter presented by counting forward three letters into the alphabet. The $S$ judged whether or not a second letter presented $1 \mathrm{sec}$ later was the correct answer. The processing demands for the letter match and ADD THREE conditions differed over the 1-sec interval between the two letter stimuli, in which Ss either merely encoded the first letter and prepared for a physical match or encoded the first letter, counted forward, and prepared for a match. The tone reaction times during the encoding period at probes 50 and $150 \mathrm{msec}$ following 
the first letter were low for both conditions. However, reaction times to probes inserted 300 and $500 \mathrm{msec}$ following the first letter were up to $600 \mathrm{msec}$ higher for the ADD THREE task than the letter match condition, indicating high processing demands for the transformation.

Utilizing tracking as a secondary task, Johnston et al (Experiments III and IV, 1970) studied the effect of different recall conditions on processing demands during a retention interval. Ss received five words and then were cued for free recall, backward recall, alphabetic recall, or ordered recall. Tracking errors were measured during an 8- or $16-\mathrm{sec}$ retention interval, in which the $S$ was presumed to "rearrange" his list when necessary. In two control conditions, Ss tracked without a primary task or while counting aloud. The distribution of tracking errors over the retention period suggested that the mental arrangement of the backward and alphabetic lists required more processing than the mere rehearsal of the free or ordered lists, while rehearsal demanded more processing than both control conditions.

Diller (1971) employed the secondary task technique, using a buttonpress whenever a visual dot pattern appeared on a screen, to determine the time course and magnitude of processing demands during an impression formation task. The $S$ received two adjectives presented serially. Following the second trait, the $\mathrm{S}$ judged either (1) whether or not the person described would be a good graduate student or (2) whether or not the person described would be likable. All traits had been prerated as either desirable or undesirable on both an academic scale and a likability scale. Sometimes one trait indicated desirability on one scale but not the other (e.g., "critical" is a desirable academic trait and an undesirable social trait). The traits were matched in four types of pairs: (1) both traits desirable or both traits undesirable on both scales (maximally similar), (2) one trait desirable and one trait undesirable on both scales (maximally dissimilar), (3) both traits desirable or undesirable on the rated scale but one trait desirable and one undesirable on the irrelevant scale, and (4) one trait desirable and one trait undesirable on the rated scale but both traits desirable or undesirable on the irrelevant scale. Diller measured the S's reaction time for making each judgment and, in addition, recorded times to note the visual probes. Probes occurred before the first trait, following onset of the first trait, or following onset of the second trait. Reaction times on the primary judgment task were longer for likability than for academic judgments. Maximally dissimilar pairs showed the longest reaction times and relevant dissimilarity and irrelevant dissimilarity, particularly for academic judgments, also produced significant effects. Reaction times to visual probes presented during the period following the first trait were lower than reaction times to probes preceding the first trait, with probe times in both these periods lower than times for probes presented following Trait 2 onset. This pattern of secondary task scores that show no interference during the encoding of the first trait and interference following the onset of the second trait mirrors the results, presented earlier, found by Posner and colleagues in letter matching tasks.

In the period following Trait-2 onset, during which impressions had to be formed, probe reaction times for traits dissimilar in relevant information were longer than times for traits similar in relevant information. Reaction times decreased over time following Trait-2 onset. These linear decreases were sharper for probes presented during maximally similar or maximally dissimilar traits. In a control condition in which Ss remembered the first trait for recall and based judgments on only the second trait, probe reaction times following second-trait presentation did not reflect differences in trait similarity. Thus, Diller's results indicated a period of high processing demands during the interval in which Ss had to evaluate traits and make a decision, with differentially higher processing demands during more difficult decisions.

\section{Responding}

Recall has been shown to cause more secondary task interference than multiple input and rehearsal (Trumbo \& Milone, 1971; Johnston et al, 1970; Johnston et al, 1972a, b; Martin, 1970). However, since secondary scores may reflect structural interference between primary and secondary responses as well as central processing demands, the studies which shed light upon the specific factors that influence processing demands are more interesting.

Word recall has been investigated for both free recall (Martin, 1970; Johnston et al, 1972b) and probed one-item semantic (category name) or positional (serial position) recall (Johnston et al, 1972a, b) with both a continuous tracking (Martin, 1970) and a discrete simple reaction-time secondary task (Johnston et al, 1972a, b). Interference with the secondary task decreases over time as more items have been recalled in free recall (Martin, 1971; Johnston et al, 1972b) and decreases over the period between the item cue and answer in probed recall (Johnston et al, 1972a). Unlike multiple-item encoding, recall is influenced by organizational factors, with processing for lists presented in categorized form lower than processing for uncategorized lists (Johnston et al. 1972b) and recall processing for blocked lists decreasing over trials more than processing for unblocked lists (Martin, 1970). In probed recall, processing demands following semantic probes are lower than demands following positional probes, with processing lower for later as opposed to early list items in both cases. This recall change across position of presentation appears to be influenced by signal to noise ratio in the presentation of material to be recalled. There is a drop in secondary task influence across position for normal high signal/noise presentation and an inverted $U$ function for low signal/noise presentation (Johnston et al, 1972a).

For learning across trials, processing demands remain 
as unclear during recall as during multiple input. Johnston et al (Experiment II, 1972b) found lowering processing demands across trials during probed recall. Martin (1970) also found a decrease across three trials in free recall, but Johnston et al (Experiment I, 1972b) found no change across 12 trials for free recall. The discrepancies between the Martin study and the Johnston et al study for both presentation and recall may reflect only the relative sensitivity of discrete and continuous secondary tasks to learning.

Noble, Trumbo, and Fowler (1967) compared processing demands during response execution, response selection, and overt learning. Ss tracked with no primary task (control) or while listening and responding verbally to a probabilistic sequence of numbers in one of four ways: (1) AR-anticipatory response of each number prior to presentation while learning the sequence, (2) FR-free response of any number prior to each presentation with no requirement to learn the sequence, (3) NR-no response while listening to the sequence in order to learn it, and (4) SR-same response as each number presented with no requirement to learn the sequence (shadowing). More errors on the secondary tracking task occurred during AR and FR than during the control condition, but there were no differences between SR, NR, and control. Decrements in AR were higher than those for FR. In other words, while the actual learning of the sequence (NR) and response execution without selection (SR) failed to demand processing, responses that required response selection and execution (AR and FR) required processing. The response (AR) requiring learning of the sequence, as well as response selection and execution, demanded more processing than the task (FR) requiring only response selection and execution. Trumbo and Noble (1970) have replicated this pattern of results using a delayed recall of CVC lists as a secondary task. The lack of interference with learning alone (NR) seems surprising but may be explained by the extended learning period without time pressure and a frequent failure of Ss to learn the sequence. Without time pressure, Ss have more freedom to decide when to rehearse a number following its occurrence. Response execution during easy shadowing tasks may be a type of response execution that does not cause interference (Greenwald, 1972; Allport et al, Experiment II, 1972). Unfortunately, while differences between processing demands during response selection and execution are important for theories of attention (e.g., Keele, 1973), these two task components remain difficult to separate within the same task under the secondary task technique.

As soon as responses that require a single movement to a target have been initiated, the processing demands during the ensuing movement depend upon the movement precision, independent of the number of initial choices of movement direction. In addition, it appears that some types of movements are automated to the extent that they do not interfere with a secondary task. Posner (reported in Posner \& Keele, 1969) compared processing demands during a wrist rotation movement that positioned a pointer on either a narrow or wide target. Ss turned the pointer to the target with the right hand and, at the same time, responded to a one-choice auditory probe with the left hand. Secondary task reaction times during movement were longer than a control condition in which Ss turned off probes while watching the $\mathrm{E}$ execute the movement. Secondary task times were significantly longer during movement to the narrow than the wide target. Ells (1969) required Ss to execute with the right hand a one-, two-, or three-choice angular movement (left, no movement, or right) to either a narrow or wide target or to a mechanical stop. As a secondary task, Ss turned off a two-choice auditory probe with the left hand whenever it occurred during the reaction-time or movement time period. During the reaction-time period between signal onset and movement initiation, the amount of time needed to turn off the probe increased with the number of movement choices but was unrelated to the degree of precision necessary for the ensuing movement. However, during the movement itself, probe reaction time depended solely upon the movement precision, with slower times for the narrow than the wide target. Movements to the stop were automated in the sense that probe reaction times in this condition were no longer than reaction times during a control condition in which Ss watched the movement. Thus, a movement that did not need to be monitored or corrected after initiation did not require processing capacity, suggesting that processing demands during movement may be related to monitoring and correction procedures.

\section{SUMMARY}

Man possesses a central system of limited capacity. There are two current theoretical positions on the nature of this limited system. The undifferentiated capacity hypothesis suggests a pool of capacity units that may be allocated to tasks as they demand processing capacity. Interference between tasks occurs when the supply of processing units is depleted. The second hypothesis proposes that some, but not all, mental operations require space in a limited capacity central mechanism. Any operation that requires space in limited capacity mechanism will interfere with any other operation that simultaneously requires space. Both hypotheses allow that different mental operations will require varying degrees of processing.

Secondary task paradigms have been employed to measure the demands that mental operations place on the limited central system. Secondary task scores may be used to compare processing demands during different mental operations within one primary task and to compare processing demands during different levels of one given primary task operation.

Two very different categories of mental operations do 
not require processing capacity and proceed in parallel with other operations without interference: (1) encoding and memory look-up as a stimulus item is received and contacts memory and (2) executing a movement to a physical stop. Other operations prove to require processing capacity. Responding, at least in recall, requires more processing capacity than multiple input, which in turn requires more processing than rehearsal. Multiple-input processing is affected by physical presentation variables such as signal/noise ratio rather than by linguistic or organizational variables, while recall and rehearsal processing demands reflect the organization and nature of the material to be recalled. Transformation tasks that require the $S$ to manipulate stimulus input in order to calculate an answer require more processing than rehearsal. Some questions, including the effect of learning on processing demands, remain unanswered.

\section{REFERENCES}

Allport, D. A., Antonis, B., \& Reynolds, P. On the division of attention: A disproof of the single channel hypothesis. Quarterly Journal of Experimental Psychology, 1972, 24, 225-235.

Bahrick, H. P., Noble, M., \& Fitts, P. M. Extratask performance as a measure of learning a primary task. Journal of Experimental Psychology, 1954, 48, 298-302.

Bertelson, P. Central intermittency twenty years later. Quarterly Journal of Experimental Psychology, 1966, 18, 153-163.

Binet, A. La concurrence des etats psychologiques. Revue Philosophique de la France et de L'etranger, 1890, 29, 138-155.

Broadbent, D. E. Perception and communication. New York: Pergamon, 1958.

Broadbent, D. E. Decision and stress. London: Academic Press, 1971.

Brooks, L. R. The suppression of visualization by reading. Quarterly Journal of Experimental Psychology, 1967, 19, 289-299.

Brown, I. D., \& Poulton, E. C. Measuring the spare "mental capacity" of car drivers by a subsidiary task. Ergonomics, $1961,4,35-40$.

Brown, J. Some tests of the decay theory of immediate memory Quarterly Journal of Experimental Psychology, 1958, 10, 12-21.

Comstock, E. M. Processing capacity in a letter-matching task Unpublished masters thesis, University of Massachusetts, 1972.

Conrad, C. E. H. Studies of the subjective lexicon. Unpublished doctoral dissertation, University of Oregon, 1972.

Crowder, R. G. Verbal short-term memory as a function of degree of learning on a perceptual-motor interpolated activity. Unpublished doctoral dissertation, University of Michigan, 1964.

Deutsch, J. A., \& Deutsch, D. Attention: Some theoretical considerations. Psychological Review, 1963, 70, 80-90.

Diller, R. D. Reaction time characteristics of an impression-formation task. Unpublished doctoral dissertation, University of Oregon, 1971.

Ells, J. G. Attentional requirements of movement control. Unpublished doctoral dissertation. University of Oregon, 1969.

Feynman, R. The character of phisical law. Cambridge, Mass: MIT Press. 1965.

Foss, D. J. Decision processes during sentence comprehension:
Effect of lexical item difficulty and position upon decision times. Journal of Verbal Learning \& Verbal Behavior, 1969, 8, $457-462$.

Foss, D. J., \& Lynch, R. H., Jr. Decision processes during sentence comprehension: Effect of lexical item difficulty and position upon decision times. Perception \& Psychophysics, $1969,5,145-148$.

Garvey, W. D., \& Taylor, F. V. Interactions among operator variables, system dynamics, and task-induced stress. Journal of Applied Psychology, 1959, 43, 79-85.

Greenwald, A. G. On doing two things at once: Time sharing as a function of ideomotor compatibility. Journal of Experimental Psychology, 1972, 94, 52-57.

James, W. The principles of psychology. New York: Holt, 1890. Jastrow, J. The interference of mental processes. American Journal of Psychology, 1891-1892, 4, 219-223.

Johnston, W. A., Greenberg, S. N., Fisher, R. P., \& Martin, D. W. Divided attention: A vehicle for monitoring memory processes. Journal of Experimental Psychology, 1970, 83, 164-171.

Johnston, W. A., Griffith, D., \& Wagstaff, R. Speed, accuracy and ease of recall. Journal of Verbal Learning \& Verbal Behavior, 1972a, 11, 512-520.

Johnston, W. A., Wagstaff, R. R., \& Griffith, D. Information processing analysis of verbal learning. Journal of Experimental Psychology, 1972b, 307-314.

Kahneman, D. Remarks on attention control. Acta Psychologica, 1970, 33, 118-131.

Kahneman, D. Attention and effort. Englewood Cliffs, N.J: Prentice-Hall, 1973, in press.

Kahneman, D., Beatty, J., \& Pollack, I. Perceptual deficit during a mental task. Science, 1967, 157, 218-219.

Karlin, L., \& Kestenbaum, R. Effects of number of alternatives on the psychological refractory period. Quarterly Journal of Experimental Psychology, 1968, 20, 167-178.

Keele, S. W. Compatibility and time-sharing in serial reaction time. Journal of Experimental Psychology, 1967, 75, 529-539.

Keele, S. W. Attention and human performance. Pacific Palisades, Calif: Goodyear Publishing, 1973.

Leonard, J. A. Tactual choice reactions. Quarterly Journal of Experimental Psychology, 1959, 11, 76-83.

Martin, D. W. Residual processing capacity during verbal organization in memory. Journal of Verbal Learning \& Verbal Behavior, 1970, 9, 391-397.

Moray, N. Where is capacity limited? A survey and a model. Acta Psychologica, 1967, 27, 84-92.

Mowbray, G. H., \& Rhoades, M. U. On the reduction of choice reaction time with practice. Quarterly Journal of Experimental Psychology, 1959, 11, 16-23.

Noble, M., Trumbo, D., \& Fowler, F. Further evidence on secondary task interference in tracking. Journal of Experimental Psychology, 1967, 79, 146-149.

Norman, D. A. Toward a theory of memory and attention. Psychological Review, 1968, 75, 522-536.

Peterson, L. R. Concurrent verbal activity. Psychological Review, 1969, 76, 376-386.

Peterson, L. R., \& Peterson, M. J. Short-term retention of individual verbal items. Journal of Experimental Psychology, $1959,58,193-198$.

Posner, M. I., \& Boies, S. J. Components of attention. Psychological Review, 1971, 78, 391-408.

Posner, M. I., \& Keele, S. W. Attention demands of movements. Proceedings of the XVIIth Congress of Applied Psycholog. Amsterdam: Zeitlinger, 1969.

Posner. M. I., \& Keele. S. W. Time and space as measures of mental operations. Invited address. Division 3. American Psychological Association, September 1970.

Posner. M. I., \& Klein, R. On the functions of consciousness. In $\mathrm{S}$. Kornblum (Ed.). Attention and performance II. Vew 
York: Academic Press, 1973. Pp. 21-35.

Posner, M. I., \& Rossman, E. Effects of size and location of informational transforms upon short-term retention. Journal of Experimental Psychology, 1965, 70, 496-505.

Savin, H. B., \& Bever, T. G. The nonperceptual reality of the phoneme. Journal of Verbal Learning \& Verbal Behavior, $1970,9,295-302$.

Seibel, R. Discrimination reaction time for a 1,023 alternative task. Journal of Experimental Psychology, 1963, 66, 215-226.

Shallice, T. Dual functions of consciousness. Psychological Review, 1972, 79, 383-393.

Shulman, H. G., \& Greenberg, S. N. Perceptual deficit due to division of attention between memory and perception. Journal of Experimental Psychology, 1971, 88, 171-176.

Shulman, H. G., Greenberg, S. N., \& Martin, J. Intertask delay as a parameter of perceptual deficit in divided attention. Journal of Experimental Psychology, 1971, 88, 439-440.

Stanners, R. F., Meunier, G. F., \& Headley, D. B. Reaction time as an index of rehearsal in short term memory. Journal of Experimental Psychology, 1969, 82, 566-570.

Trumbo, D., \& Milone, F. Primary task performance as a function of encoding, retention, and recall in a secondary task. Journal of Experimental Psychology, 1971, 91, 272-279.

Trumbo, D., \& Noble, M. Secondary task effect on serial verbal leaming. Journal of Experimental Psychology, 1970, 85, 418-424.

Warren, R. E. Stimulus encoding and memory. Journal of
Experimental Psychology, 1972, 94, 90-100

Welch, J. C. On the measurement of mental activity through muscular activity and the determination of a constant attention. American Journal of Physiology, 1898, 1, 283-306.

Welford, A. T. The "psychological refractory period" and the timing of high speed performance: A review and a theory. British Journal of Psychology, 1952, 43, 2-19.

Welford, A. T. Fundamentals of skill. London: Methuen, 1968

\section{NOTES}

1. Task interference techniques allow task demands on processing space to be compared along relative scales. It is also possible that physiological indices such as pupil diameter will provide meaningful common units for comparing different tasks (Kahneman, 1973).

2. The nature of the task to be performed determines the extent of available capacity. Thus, "one cannot work as hard in retaining four digits as one must work to complete a mental multiplication of two-digit numbers [Kahneman, 1973, Chapter II]." A nonlinear response to increasing demand leads to increasing discrepancy between the effort that a task demands and the amount actually supplied

(Received for publication January 31, 1973; revision received March 21, 1973.) 